\title{
Metodología de docencia inversa online: herramientas utilizadas y aplicación a la asignatura de redes eléctricas inteligentes durante el aislamiento debido al estado de alarma producido por la COVID-19
}

\author{
Carlos Varga-Salgado ${ }^{a}$, Paula Bastida-Molina ${ }^{\mathrm{b}}$, Manuel Alcazar-Ortega ${ }^{\mathrm{c}}$ y Lina Montuori $^{\mathrm{d}}$
}

${ }^{a}$ Departamento de Ingeniería Eléctrica, Camino de Vera s/n Edificio 5E, Universitat Politècnica de València, España, carvarsa@upvnet.upv.es, ${ }^{b}$ Instituto Universitario de Ingeniería Energética, Camino de Vera s/n, Edificio 8E, 2a planta, Universitat Politècnica de València, España, paubasmo@etsid.upv.es, ${ }^{\circ}$ Departamento de Ingeniería Eléctrica, Camino de Vera, s/n Edificio 5E, Universitat Politècnica de València, España, malcazar@iiie.upv.es, ${ }^{\mathrm{d}}$ Departamento de Termodinámica Aplicada, Universitat Politècnica de València, Camino de Vera, s/n, edificio 5J, $2^{\mathrm{a}}$ planta, 46022 Valencia (España), lmontuori@upvnet.upv.es

\begin{abstract}
In this paper, the Flipped-teaching methodology used in the Polytechnic University of Valencia is applied to the Smartgrids course of the master's degree in Energy Technology for Sustainable Development. The use of this methodology has interest due to the crises caused by the COVID-19 virus and the change of classroom classes to non-classroom classes. According to the procedure, the Flipped-teaching methodology is presented to the student beside to the work plan. Both synchronous and asynchronous tools have been used in this methodology. The synchronous tools used are MS Teams and skype and among the asynchronous ones are MS PowertPoint, Poliformat, OBS studio and MSRecorder. Media UPV and YouTube have been used to spread the videos. To work in a friendly environment. Lessons, which is a tool integrated in PoliformaT, has been used. As a result, it can be highlighted that, the average final mark is similar in both courses: online and face-to-face. Finally, although the effort to mount the course the first year, the learning task is facilitated for the student and the professor can take profit the work in the following years.
\end{abstract}

Keywords: Flipped-teaching methodology, skills, training, Online teaching

\section{Resumen}

En este paper se aplica la metodología de docencia inversa utilizada en la UPV, a la asignatura de Redes Eléctricas Inteligentes. Tiene especial interés dicha metodología debido a la pandemia producida por COVID-19 y el cambio de actividad académica de presencial a no presencial en la $U P V$. De acuerdo con el procedimiento, se realiza la presentación de la asignatura, se explica la metodología y se presenta el plan de trabajo al estudiante. Se han utilizado herramientas tanto síncronas como asíncronas. Las herramientas síncronas utilizadas son MS Teams y Skype y entre las asincronas se encuentran MS PowerPoint, PoliformaT, OBS studio MSRecorder. Para difundir los videos se ha utilizado Media UPV. Para que la enseñanza sea más interactiva y con un entorno más agradable se ha utilizado Lessons, (herramienta integrada en PoliformaT). Como resultados destaca la comparación de los resultados de la evaluación de las clase presenciales y online. Las notas medias obtenidas son similares. Se ha utilizado un método de evaluación muy similar al de las clases tradicionales. Destaca además el elevado esfuerzo de montar la asignatura el primer año, pero facilita la tarea de aprendizaje al alumno y se puede amortizar el trabajo durante los siguientes años.

Palabras clave: docencia inversa, competencias, formación, metodología, docencia online. 


\section{Introducción}

Debido al estado de alarma producido en España en marzo de 2020 por la expansión de la pandemia de provocada por la enfermedad COVID-19 y producida por el virus SARS-CoV-2, el país entero prácticamente se paraliza, permitiendo solo la operación de los servicios imprescindibles para cubrir las necesidades de todo el país. Debido a la prohibición de reuniones y actividades grupales y al no ser la enseñanza un servicio imprescindible en momentos de emergencia, las instituciones educativas que no lo habían hecho, paran sus actividades académicas antes del 15 de marzo de 2020, día en que empieza a regir dicho estado de alarma, por tanto, las universidades españolas, incluida la UPV, suspenden la actividad académica presencial. En el caso de la UPV, y según acuerdo con otras universidades y con la Conselleria de educación de la Generalitat Valenciana, cultura y deporte de la Comunidad Valenciana, se decide seguir el curso universitario de forma Online (Figura 1).

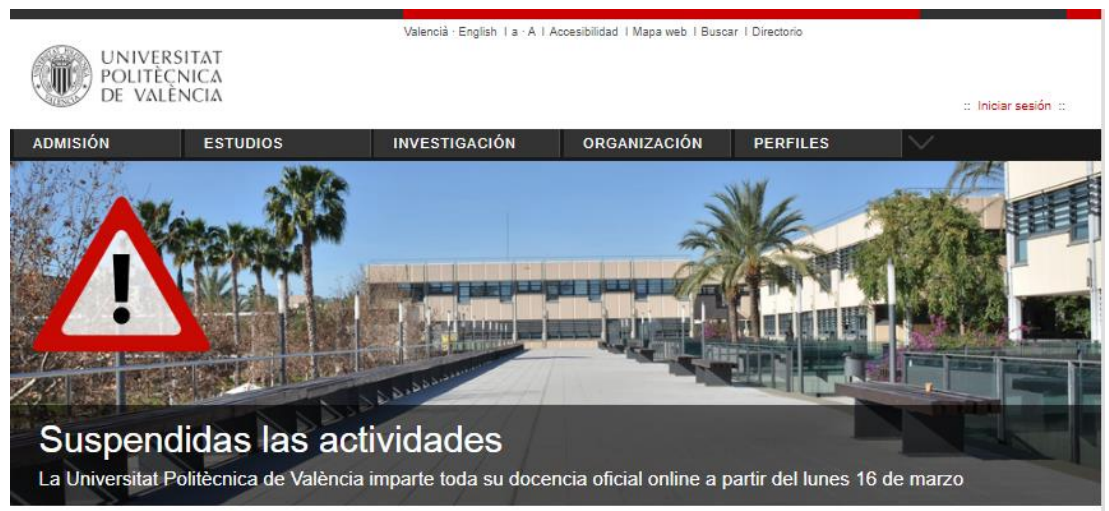

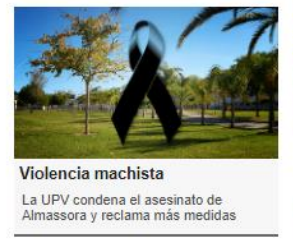

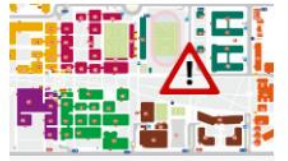

Parkings cerrados en el campus El acceso a la UPV está limitado solo al personal autorizado
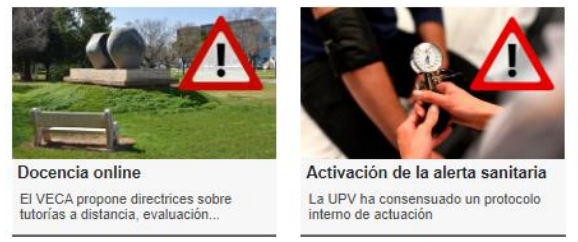

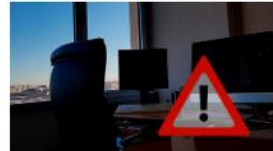

Nuevas medidas excepcionales La úttima resolución del rector modifica. el funcionamiento de la UPV

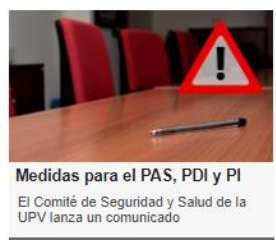

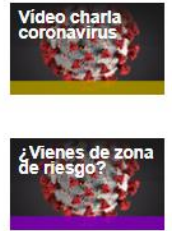
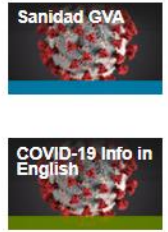

Fig. 1. Página web de la UPV referida a la docencia online debida a la COVID-19.

Por otro lado, la segunda parte de la asignatura Redes eléctricas inteligentes del Master universitario en Tecnología energética para el desarrollo Sostenible (MUTEDS) de la Escuela Técnica Superior de Ingenieria Industrial (ETSII) de UPV, con 16 estudiantes matriculados (de los cueles 15 acabaron el curso y realizaron todos los actos de evaluación), se iba realizar aplicando docencia inversa, pero debido a los importantes cambios en la metodología de la enseñanza en la UPV por no ser la clase presenciales, se ha modificado el procedimiento para incluir herramientas síncronas para impartir las clases, reforzando el uso de las herramientas asíncronas de aprendizaje.

Para aplicar el método de docencia inversa se mantendrá la esencia de la metodología aprovechando su fortaleza en cursos online. Por tanto, se aplicarán el método de metodología inversa de la UPV combinándolo con las recomendaciones de la UPV para impartir la docencia online. 


\section{Objetivos}

El objetivo de este trabajo es analizar y comparar con el curso anterior los resultados de la evaluación, aplicando la metodología de docencia inversa Online a la asignatura de Redes Eléctricas Inteligentes del Máster Universitario de Tecnología Energética para el Desarrollo Sostenible de la UPV. Esta metodología se aplicará durante la impartición de docencia oficial online debido al estado de alarma provocado por enfermedad COVID-19.

\section{Desarrollo de la innovación}

La metodología de docencia inversa consiste el refuerzo de la enseñanza del alumno partiendo de la premisa de que si el alumno prepara la clase desde casa y en las clases (en este caso virtuales) solo resuelve las dudas y realiza los problemas planteados, el aprendizaje sería más efectivo. El alumno debe estudiar de forma autónoma, antes de cada sesión de clase, los temas de aprendizaje más sencillos, apoyado en la bibliografía y videos recomendados por el profesor, además del material propio preparado también por el profesor. Posteriormente, en la sesión de clase online, el estudiante trabaja los temas de mayor complejidad con la ayuda del profesor y el trabajo en equipos con sus compañeros, posteriormente y una vez acabada la clase, el estudiante repasa y asimila los conceptos más complejos vistos durante la sesión.

\subsection{Metodología}

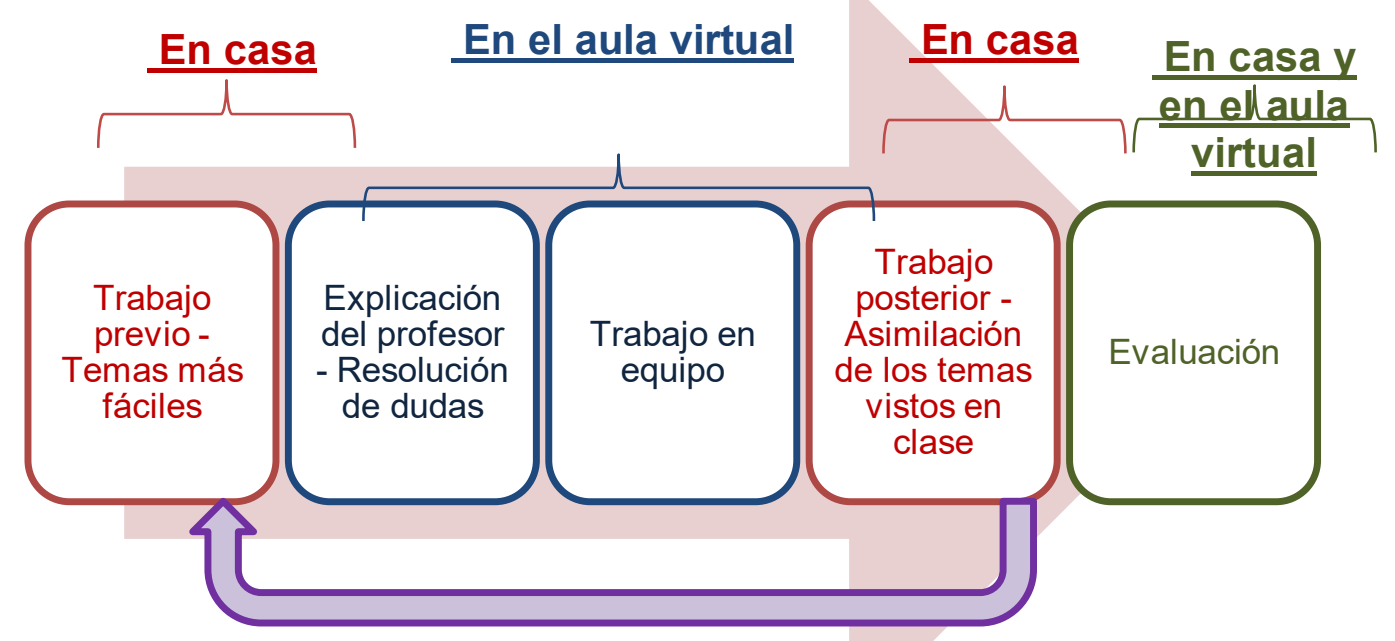

\section{Este bucle se repite hasta la última sesión}

Fig. 2. Metodología de docencia inversa aplicada a la asignatura.

Para aplicar de la metodología, se ha utilizado la herramienta Lessons, junto con el resto de herramientas de PoliformaT disponibles en la UPV, pero también se podrían utilizar otras plataformas de uso libre como Google classroom. En la primera clase se presenta la metodología a los estudiantes. En dicha metodología se deja claro que el estudiante ha de preparar la clase previamente en casa, según las indicaciones del profesor y posteriormente en la sesión se aclararán las dudas surgidas durante la preparación de los temas. Se han realizado además animaciones y explicaciones con detalle de los conceptos más difíciles de asimilar. Los estudiantes, mediante el trabajo en grupo, deben llevar a cabo un proyecto con el que se evaluará la asignatura. Los grupos realizan avances semanales en el proyecto, integrando la parte vista en cada sesión, dicha sesión coincide con la parte del trabajo en la que el grupo tiene que avanzar. Además se deja claro en el método y el peso de cada evaluación. La evaluación se dividirá en cuatro partes: trabajo en clase, 
presentación del proyecto, defensa del proyecto y test realizados mediante la aplicación exámenes de PoliformaT. La figura 2 muestra la metodología de docencia inversa aplicada a la asignatura.

\subsection{Herramientas utilizadas}

Las herramientas utilizadas se han clasificado como herramientas síncronas y herramientas asíncronas. Estas herramientas pueden ser gratuitas, propias de la UPV o la UPV dispone de la licencia.

\subsubsection{Herramientas para docencia síncrona}

Son aquellas herramientas virtuales que permiten una interacción directa y en tiempo "real" entre el profesor y los estudiantes. Mediante estas herramientas se pueden conservar los horarios habituales de clase. Para aplicar esta metodología se utilizan: Teams y Skype. En términos generales ambas aplicaciones permiten realizar reuniones virtuales, chats, intercambio de archivos y compartir pantalla. La figura 2 muestras las herramientas síncronas utilizadas para la docencia inversa en la asignatura Redes Eléctricas Inteligentes. La figura 3 muestra las herramientas MS Teams y Skype. A continuación, se explican dichas herramientas.

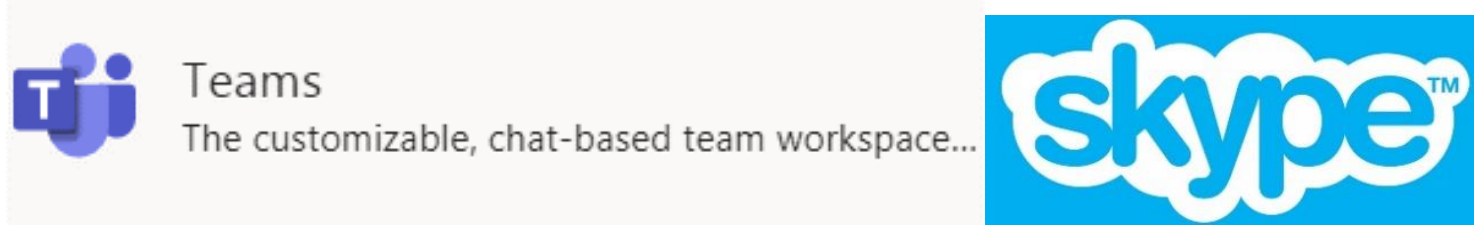

Fig. 3. Herramientas síncronas utilizadas para la docencia inversa en la asignatura de redes inteligentes.

\section{Microsoft Teams}

Es una herramienta de Microsoft integrada en el paquete de office 365, para su uso hace falta adquirir la licencia. La UPV dispone de dicha licencia, por tanto, puede ser utilizada por toda la comunidad universitaria. En esta metodología la herramienta se utiliza para docencia virtual online de forma síncrona y para las tutorías. Como ventajas a destacar tiene que el número de usuarios que pueden hacer parte de una reunión es elevado (500 conexiones al mismo tiempo). Además tiene herramientas interesantes para dar las clases tales como que permite silenciar a el resto de participantes con un clic, permite que un miembro de la reunión pueda tomar el control de la pantalla compartida, además permite hacer grupos de estudiantes, de tal forma que estos pueden reunirse de forma independiente y el profesor puede entrar a cualquiera de las reuniones de los grupos y hacer aclaraciones o resolver dudas. Estas opciones pueden ser personalizadas por el profesor. Tiene otras propiedades como difuminar el fondo de la pantalla, útil para que no se vea el fondo del sitio donde estamos y además permite grabar la clase, por lo tanto, los estudiantes disponen del video para repasar la parte que le interese de la clase.

\section{Skype}

La versión gratuita Skype permite hasta 50 conexiones al mismo tiempo, por tanto, para clases con más de 50 estudiantes no es viable. Como ventaja se podría mencionar que es una aplicación gratuita y que es una de las más conocidas y utilizada. En este contexto solo se utilizará para tutorías con los estudiantes.

\subsubsection{Herramientas para docencia asíncronas}

Las herramientas para docencia asíncronas permiten poner a disposición de los estudiantes el material de clases, de tal manera que puede ser consultado en cualquier momento. El material podría incluir videos, libros, presentaciones, etc. Se utilizarán herramientas asíncronas propias de la UPV, externas gratuitas y externas de las cuales la UPV dispone de la licencia. A continuación, se explican las herramientas asíncronas utilizadas en esta metodología: 


\section{Herramienta para compartir documentos y realizar anuncios - PoliformaT}

La plataforma universal asíncrona de la UPV es PoliformaT. Tanto el profesorado como el alumnado está familiarizados con el uso de PoliformaT por tanto se utiliza esta herramienta para compartir documentos, hacer convocatorias, enviar mensajes a los estudiantes, realizar test de examen, etc. Además, dentro de PoliformaT se encuentra la herramienta Lessons, que se utiliza para crear y acceder a los contenidos de una manera cómoda y visualmente interesante. En la figura 4 se muestran las herramientas asíncronas para compartir documentos utilizadas en esta metodología.

\section{PoliformaT}

Es la plataforma de teleformación donde los alumnos y profesores comparten la información.

\section{LESSONS}

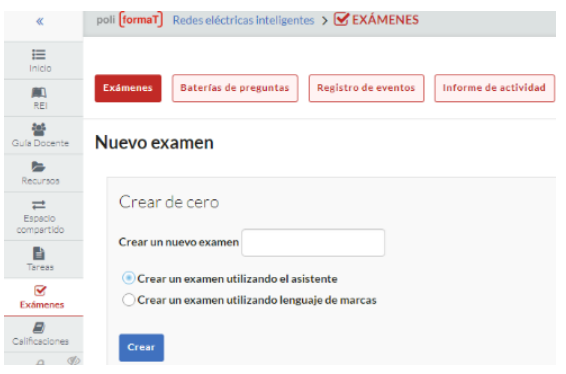

Fig. 4. Herramientas para compartir documentos y crear exámenes: PoliformaT y dentro de PoliformaT Lessons y exámenes.

\section{Herramientas para grabar pantalla o presentaciones (Screencast)}

Las herramientas utilizadas en esta metodología para realizar videos son PowerPoint, que es una herramienta de pago integrada en office 365 , además también se utilizan las herramientas gratuitas OBS y SMRecorder.

PowerPoint

Es la herramienta más difundida para realizar presentaciones en la comunidad universitaria. Además se pueden realizar videos de una presentación de forma muy fácil, para ello la versión 2016 cuenta con 2 opciones:

- Realizar la narración sobre la misma presentación utilizando la función grabar presentación que se muestra en la figura 5, Una vez acabada la presentación se puede enviar a los estudiantes en formato pptx de PowerPoint, PDF o guardar la presentación en formato de video mp4. Como ventajas destacan que el material multimedia se asocia a cada diapositiva de forma independiente, por tanto, se puede borrar lo comentado en cada diapositiva de forma independiente, evitando repetir toda la grabación. Como desventaja se encuentra que solo se puede grabar la presentación de PowerPoint y no la pantalla de tal forma de que si se quiere visualizar algo distinto, como por ejemplo una página web, no sería fácilmente posible de una forma sencilla. Además, el peso del video, una vez se ha convertido a formato mp4 es elevado.

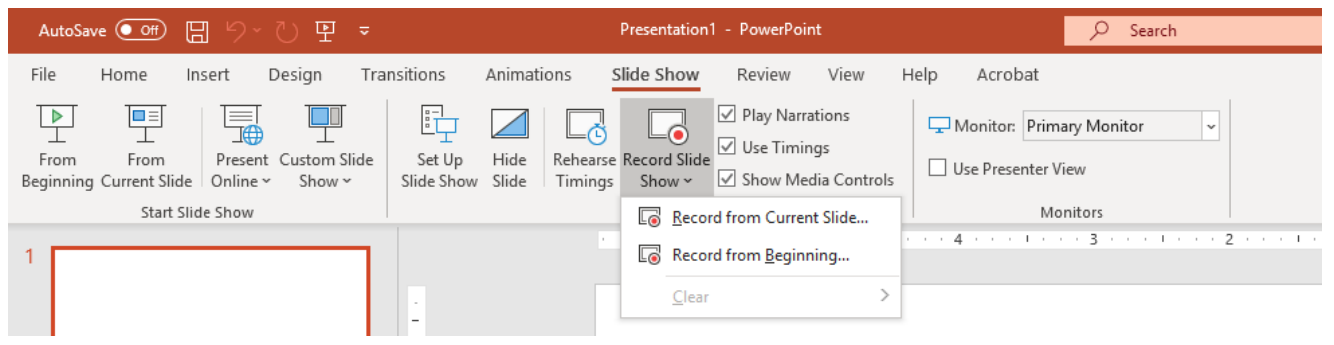

Fig. 5. Grabar presentación en PowerPoint 
- Realizar la grabación de la pantalla completa o de una parte de ella, PowerPoint 2016 también tiene la opción de grabar la pantalla (Screencast), Una vez finaliza la grabación veremos una imagen en la diapositiva, que en modo presentación visualizaría el video, Haciendo clic derecho y guardando el archivo como media se podría convertir a un archivo mp4. La figura 6 muestra la opción de grabar pantalla en el menú insertar.

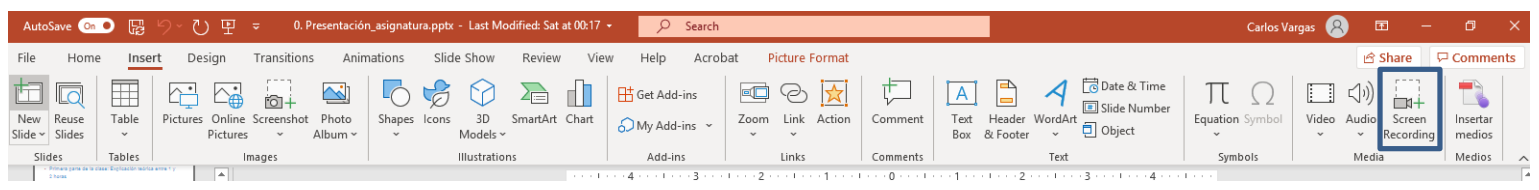

Fig. 6, Grabar pantalla con PowerPoint.

SMRecorder

Permite realizar grabaciones de pantalla de forma muy simple y seleccionando la parte de la pantalla que nos interesa grabar de forma muy parecida a como lo hace PowerPoint utilizando screencast. La figura 7 muestra una pantalla de SMRecorder.

\section{四 SMRecorder 1.3.2

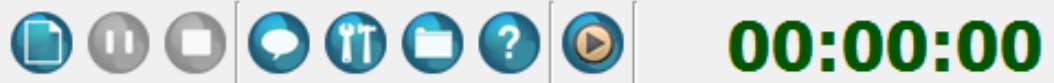

Fig. 7. Grabar pantalla con SMRecorder.

OBS studio

Programa gratuito para realizar screncast. El programa funciona por medio de "Escenas" que se componen usando "fuentes". Ya que es gratuito, es muy utilizado por youtubers y videogamers. Es más complejo de utilizar que la aplicación de PowerPoint o SMRecorder, pero también es más versátil. Tiene la opción de retrasmitir en streaming. La figura 8 muestra una pantalla de OBS studio.

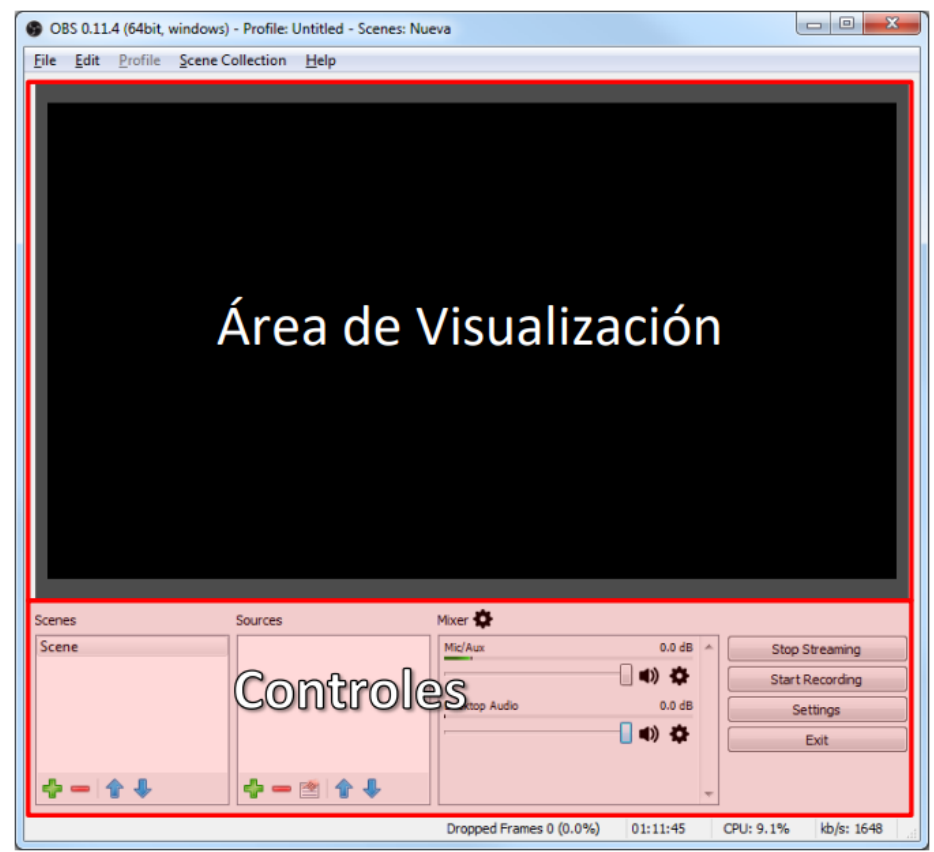

Fig. 8. Pantalla con $O B S$ 


\section{Herramientas para divulgación de los videos}

Para divulgar los videos se utilizarán dos opciones: media UPV y YouTube, Media UPV es una aplicación propia de la UPV para compartir videos, los videos se pueden dejar ocultos de tal forma que para verlos se requiere del link. Para subir videos hace falta ser parte de la comunidad universitaria. Los videos pueden incrustarse en Lessons o también se puede compartir el link con los estudiantes. La figura 9 muestra una pantalla de media UPV, Youtube es la aplicación universal para compartir videos (Figura 10).

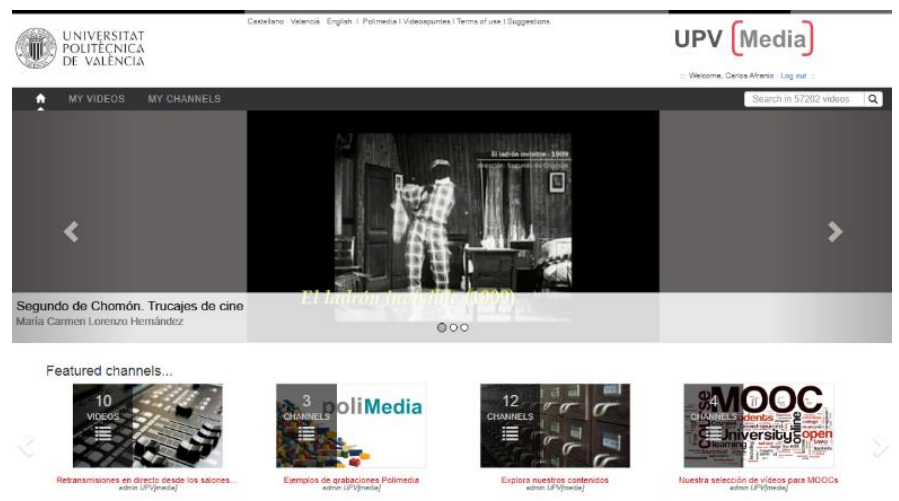

Fig. 9. Pantalla de media UPV

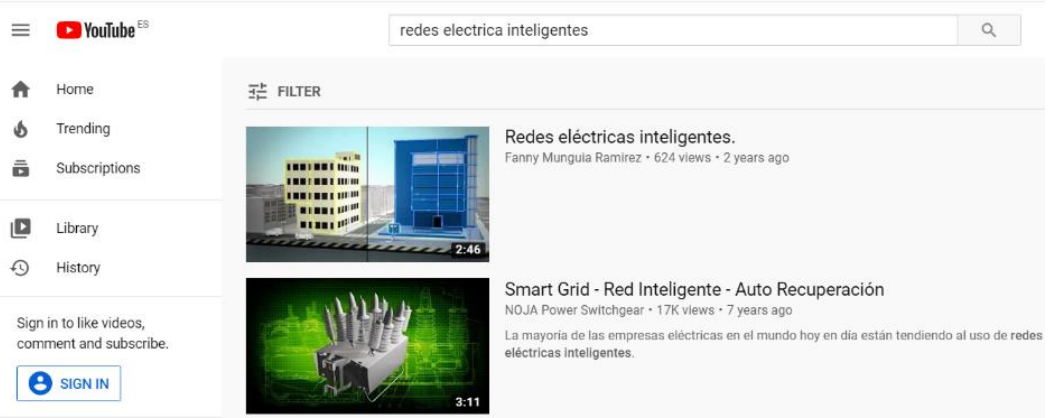

Fig. 10. Pantalla de YouTube

\section{Evaluación}

La evaluación de la segunda parte de la asignatura, equivalente al $50 \%$ de la nota total, se lleva a cabo mediante una prueba tipo test y un proyecto que llevan a cabo lo estudiantes. El peso de cada evaluación se muestra en la tabla 1 :

Tabla 1. Actos de evaluación de asignatura Redes Eléctricas Inteligentes.

\begin{tabular}{|l|l|l|}
\hline \multicolumn{2}{|c|}{ Acto de evaluación } & Peso \\
\hline \multirow{3}{*}{ Test } & Informes semanales & $30 \%$ \\
\cline { 2 - 3 } & Informe final & $10 \%$ \\
\cline { 2 - 3 } & Defensa individual (exposición) & $20 \%$ \\
\hline
\end{tabular}

La prueba incluye preguntas de teoría y cálculos rápidos. El proyecto consta de informes semanales que se envían al profesor una vez realizados los avances indicados en el plan de trabajo, una memoria técnica del proyecto, que trata del diseño de una microrred eléctrica inteligente, y de la defensa del proyecto de forma individual en la que el estudiante realiza una presentación y el profesor hace preguntas. 
Metodología de docencia inversa online: herramientas utilizadas y aplicación a la asignatura de redes eléctricas inteligentes durante el estado de alarma producido por la COVID-19

\section{Resultados}

A continuación, se muestran los resultados de la metodología. Los resultados se dividen en material de soporte desarrollo para llevar a cabo la metodología y resultados de la aplicación de la metodología.

\section{Material de soporte desarrollo para llevar a cabo la metodología}

El menú de Lesson se muestra en la Figura 10.

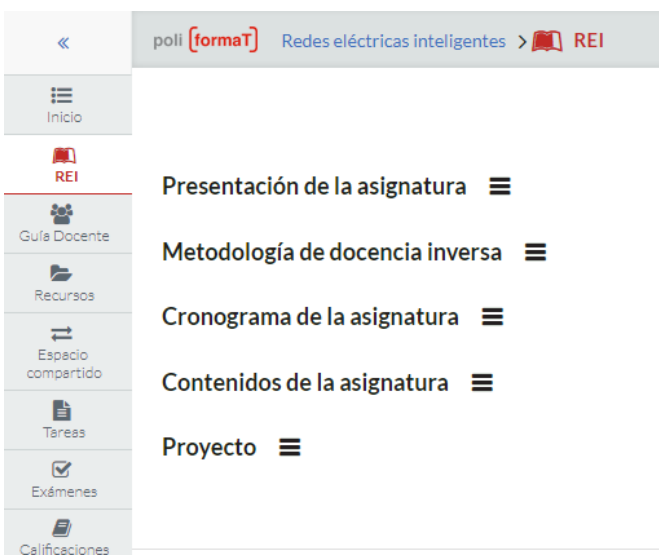

Fig. 11. Menú de Lessons para acceder a las distintas partes de la asignatura

La bienvenida a la asignatura se muestra en la figura 11.

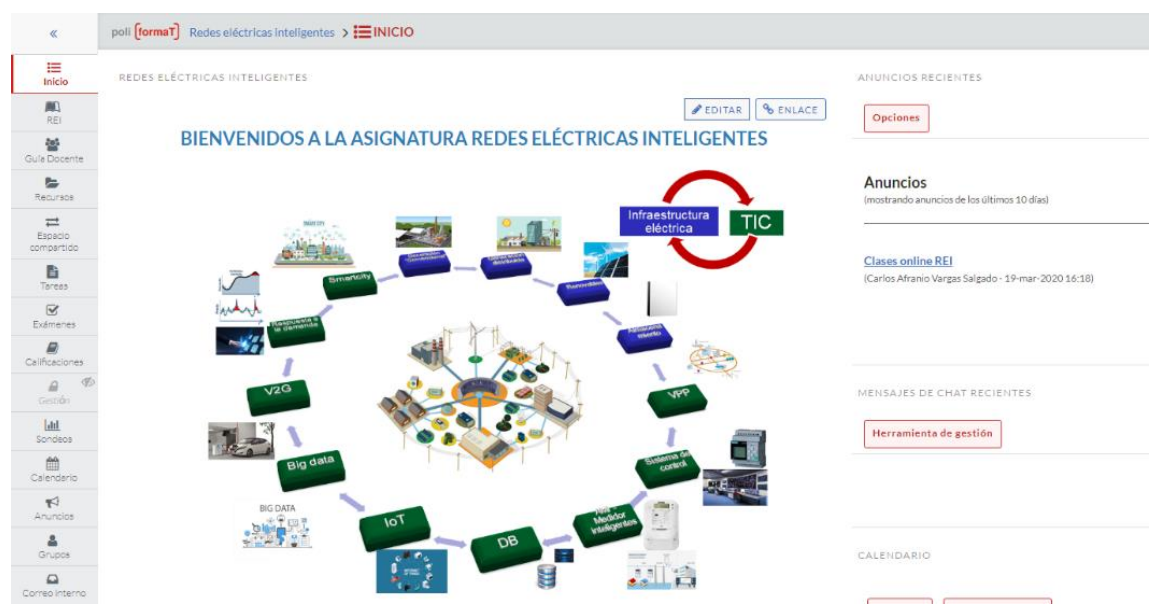

Fig. 11. Bienvenida a la asignatura

El enlace para acceder a la presentación de las asignaturas se muestra en la figura 12.

\section{Presentación de la asignatura}
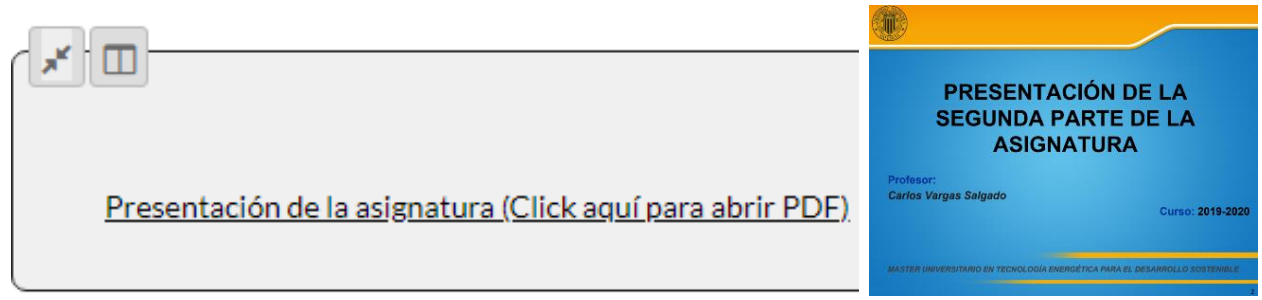

Fig. 12. Enlace para abrir un PDF con la presentación de la asignatura (a la derecha).

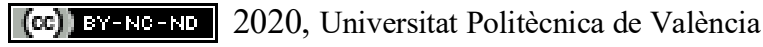

Congreso In-Red (2020) 
La explicación de la metodología de docencia inversa se muestra en la figura 13.

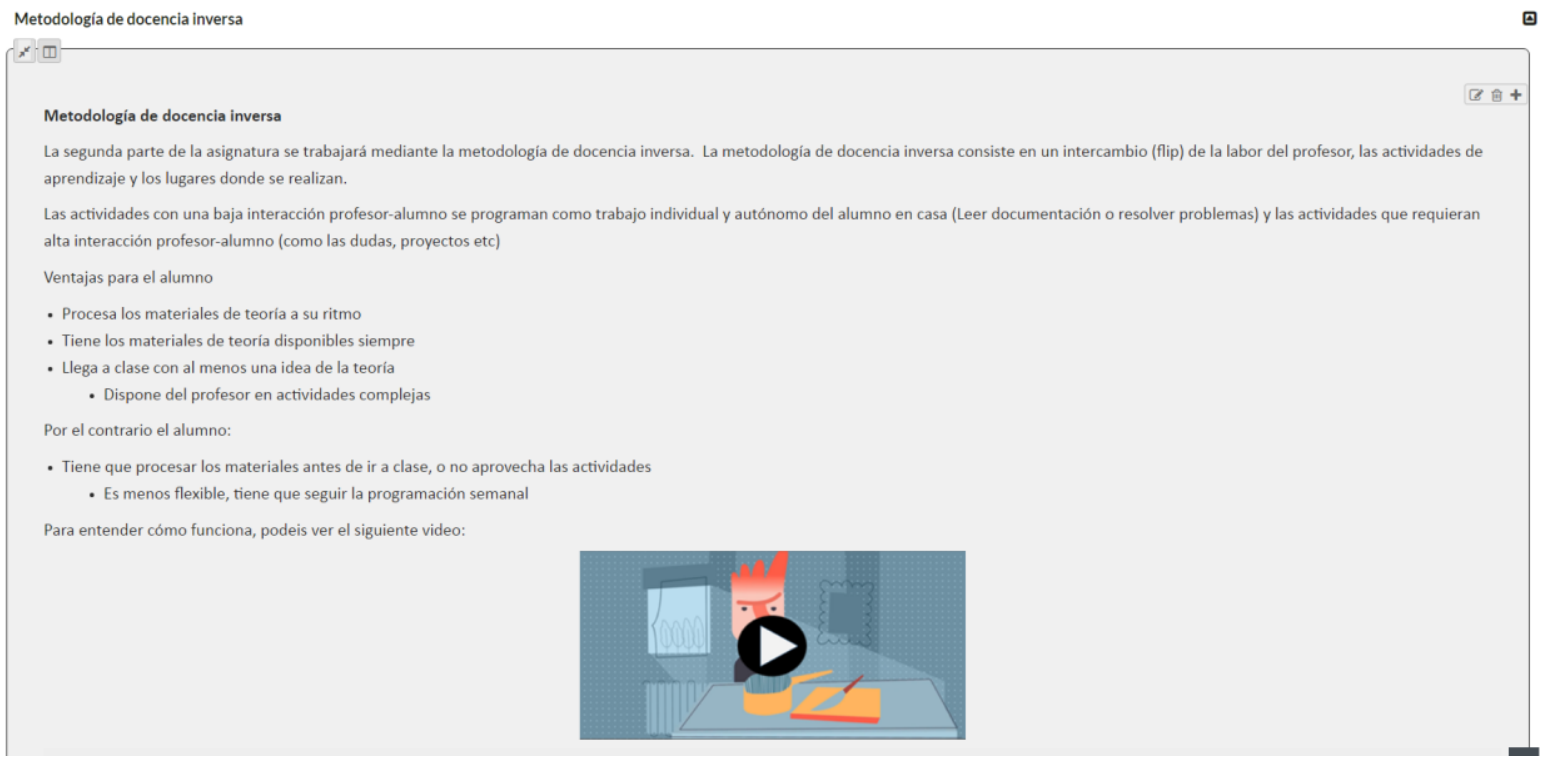

Fig. 13. Explicación de la metodología de docencia inversa.

La presentación del plan de trabajo de las dos primeras semanas se muestra en la figura 14.

$\underline{\text { SEMANA } 1 \text { y } 2 \text {-[Clases lunes] }}$

\begin{tabular}{|c|c|c|c|c|}
\hline & $\begin{array}{l}\text { RESULTADO DE } \\
\text { APRENDIZAJE }\end{array}$ & TRABAJO PREVIO EN CASA & $\begin{array}{l}\text { TRABAJO EN EL } \\
\text { AULA }\end{array}$ & $\begin{array}{c}\text { TRABAJO } \\
\text { POSTERIOR EN CASA }\end{array}$ \\
\hline $\begin{array}{c}\text { S1: LUNES } \\
23 / 03 / 2020\end{array}$ & $\begin{array}{l}\text { Definir el concepto de red } \\
\text { eléctrica inteligente (REI). } \\
\text { Identificar a los entes que } \\
\text { gestionan una REI } \\
\text { Identificar las caracteristicas } \\
\text { de una REI. } \\
\text { Enumerar las barreras de } \\
\text { una red eléctrica inteligente. }\end{array}$ & $\begin{array}{l}\text { Leer y analizar presentación de la sesión } 1 \\
\text { Visualización del video "La smart grid" (1'14"): } \\
\text { https://www.youtube.com/watch? } \\
\underline{\text { v=Eb5tUcvbpLM\&feature=youtu.be }}\end{array}$ & $\begin{array}{l}\text { Explicación de la asignatura (Temas, } \\
\text { metodologia, proyecto, presentaciones y } \\
\text { evaluación) } \\
\text { Organización de los grupos de trabajo y } \\
\text { explicación del proyecto a realizar } \\
\text { Aplicando los conceptos aprendidos, } \\
\text { empezar trabajo de la asignatura. }\end{array}$ & $\begin{array}{l}\text { Acabar con los } \\
\text { objeticos del } \\
\text { proyectos planteados } \\
\text { para la sesión. } \\
\text { Preparar la siguiente } \\
\text { sesión. }\end{array}$ \\
\hline $\begin{array}{l}\text { S2: LUNES } \\
30 / 03 / 2020\end{array}$ & $\begin{array}{l}\text { Clasificar los componentes } \\
\text { de una REI y la función de } \\
\text { cada uno. } \\
\text { Definir VPP, Generación } \\
\text { distribuida, Control en REI. } \\
\text { Identificar los equipos de } \\
\text { medida, AMI, smartmeter, } \\
\text { cables de comunicaciones. }\end{array}$ & $\begin{array}{l}\text { Leer y analizar presentación de la sesión } 2 \\
\text { Ver videos: } \\
\text { Virtual Power Plant (VPP), a new form of energy } \\
\text { management }\left(1^{\prime} 45^{\prime \prime}\right) \text { : } \\
\text { https://www.youtube.com/watch?v=7/bZF5ZOpZ4 } \\
\text { AMI \& Smart Meter Solutions (1'48") } \\
\text { https://www.youtube.com/watch?v=rOD_bHcOSjo }\end{array}$ & $\begin{array}{l}\text { Aplicando los conceptos aprendidos, } \\
\text { empezar trabajo de la asignatura. }\end{array}$ & $\begin{array}{l}\text { Acabar con los } \\
\text { objeticos del } \\
\text { proyectos planteados } \\
\text { para la sesión. } \\
\text { Preparar la siguiente } \\
\text { sesión. }\end{array}$ \\
\hline
\end{tabular}

Fig. 14. Plan de trabajo de las dos primeras ras semanas. 
Metodología de docencia inversa online: herramientas utilizadas y aplicación a la asignatura de redes eléctricas inteligentes durante el estado de alarma producido por la COVID-19

Los contenidos de la asignatura se muestran en la figura 15.

$\square$

\section{Contenidos de la asignatura}

Los temas de la segunda parte de la asignatura se realizarán en 6 sesiones entre el 23 de marzo y el 1 de junio Los temas a tratar son los siguientes:

\section{Sesión 1 (Click aquíp para ver contenido).}

5. Tema 5: Redes inteligentes

5.1. Introducción

5.2. Pasado y presente.

5.3. Gestión

5.4. Concepto

5.5. Características

5.6. Barreras

Sesión 2 (Click aquí para ver contenido).

5.7. Componentes de una red eléctrica inteligente

5.7.1. VPP, Generación distribuida

5.7.2. Control

5.7.3. Equipos de medida

5.7.4. AMI, Smartmeter

Fig. 15. Contenido de la asignatura.

El enlace para acceder al PDF con la explicación del proyecto a realizar por los estudiantes se muestra en la figura 16.

Proyecto

$\pi-\square$

Proyecto REI (Click aquípara ver contenido).

Realizar una propuesta de un proyecto piloto para convertir una red eléctrica convencional de una población de la provincia de Valencia en una red eléctrica inteligente.

El proyecto debe realizarse:

-En grupos de 4 estudiantes (Aprox)

-Se debe definir e implementar las necesidades para el desarrollo del proyecto:

I. Recolectar información

II. Análisis y sintesis de como abordar el proyecto.

III. Desarrollo del proyecto.

IV. Presentación de los resultados en un trabajo que se debe entregar el día de la presentación.

V. Defensa del trabaja mediante una presentación.

Fig. 16. Enlace para acceder al PDF que muestra el proyecto a realizar por los estudiantes en la asignatura.

La convocatoria para una clase síncrona mediante Teams se muestran en la figura 17.

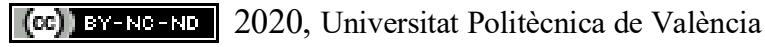

Congreso In-Red (2020) 


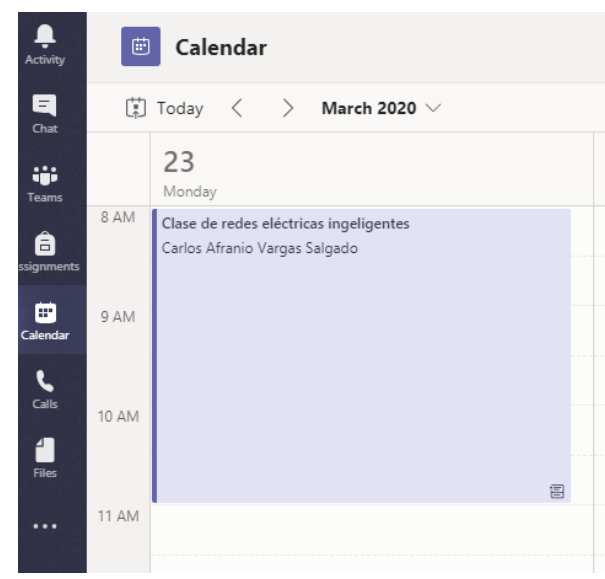

Fig. 17. Convocatoria para una clase online a través de MS Teams el día 23 de marzo.

\section{Resultados de la aplicación de la metodología - Evaluación y comparativa con el curso anterior}

Después de aplicar la metodología se ha comparado el resultado de las evaluaciones de ambos cursos. El tipo de evaluación se realizó de la forma más similar posible comparado con la evaluación presencial. El número de estudiantes evaluados en 2019 fue de 17 y en 2020 había 16 matriculados, de los cuales 15 realizaron todos los actos de evaluación.

Tabla 2. Comparativa de las evaluaciones realizadas en el 2019 mediante el método tradicional y en el 2020 mediante docencia online.

\begin{tabular}{|l|c|c|c|c|c|c|}
\hline \multicolumn{1}{|c}{ Nota } & Test & Proyecto & Total & \multicolumn{4}{c|}{ Test } & \multicolumn{2}{c|}{ Proyecto } & \multicolumn{1}{c|}{ Total } \\
\hline Promedio & 7,4 & 9,1 & $\mathbf{8 , 6}$ & 7,1 & 9,0 & $\mathbf{8 , 4}$ \\
\hline Mínima & 5,0 & 8,6 & $\mathbf{7 , 6}$ & 2,6 & 8,5 & $\mathbf{6 , 8}$ \\
\hline Máxima & 10,0 & 9,5 & $\mathbf{9 , 7}$ & 10,0 & 9,4 & $\mathbf{9 , 4}$ \\
\hline
\end{tabular}

Los resultados de la comparativa de las evaluaciones realizadas en 2019 y en 2020 se muestra en la tabla 2. Los resultados obtenidos son muy similares, siendo la nota media 8,6 en 2019 y 8,4 en 2020. En la figura 18 (izquierda) se observa la nota final de todos los estudiantes en 2019 y en 2020 ordenada de mayor a menor. De este gráfico se deduce que las notas fueron ligeramente superiores en el año 2019 comparada con el año 2020. En la misma figura 18 (derecha) se observa que la calificación más frecuente es notable con un $71 \%$ en 2019 y un $73 \%$ en 2020 .
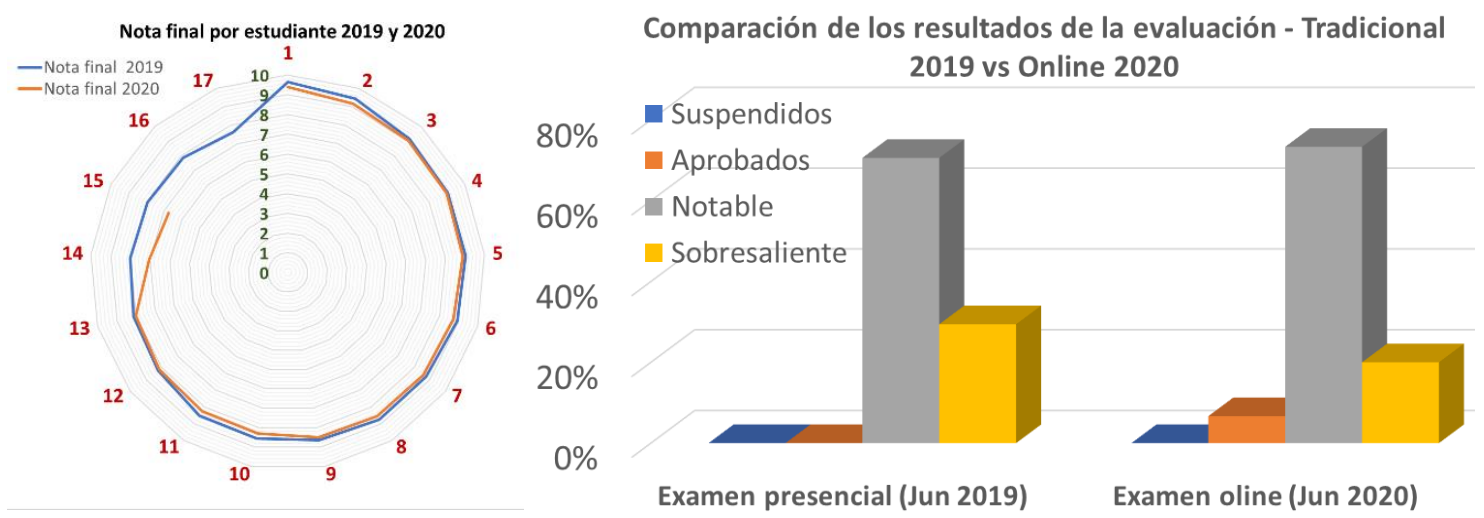

Fig. 18. Comparación de los resultados del examen presencial (2019) y online (2020). 


\section{Conclusiones}

En la actualidad existen múltiples herramientas que permiten llevar a cabo la docencia Online. Ya que no hay una herramienta que lo integre todo, se debe recurrir a múltiples aplicaciones. Si no se han utilizado nunca algunas de estas aplicaciones, podría llevar esfuerzo y tiempo en escogerlas y aprender a utilizarlas. Mediante este artículo se expone la experiencia del uso de varias herramientas que permiten realizar docencia tanto síncrona como asíncrona, muchas de ellas de uso libre, permitiendo ahorrar tiempo en la selección de dichas herramientas.

Por otra parte, se ha realizado un plan de trabajo y se ha centralizado toda la documentación del curso utilizando la herramienta Lessons integrada en la plataforma de PoliformaT. Aunque no se ha realizado ninguna encuesta que permita cuantificar el grado de satisfacción del estudiante, en general los estudiantes encontraban muy útil poder tener toda la información organizada antes de empezar el curso y disponer de los videos después de cada sección. El numero de consultas y tutorías al profesor también disminuyó considerablemente, probablemente debido que se disponía de toda la información.

Finalmente se realizado la evaluación en el 2020 de la forma más parecida posible en los cursos presenciales del año 2019 y se han comparado los resultados de ambas evaluaciones. Se puede concluir que con la metodología aplicada los resultados de la evaluación son muy similares, obteniendo una nota media de 8,6 y 8,4 en las evaluaciones de los años 2019 y 2020 respectivamente. De igual forma las notas medias de los exámenes (7,4 y 7,1 en 2019 y 2020) y de los proyectos realizados ( 9,1 y 9 en 2019 y 2020) es muy similar también. Se deduce que la metodología de docencia inversa online, tienes resultados muy parecidos en las evaluaciones tanto presenciales como online.

\section{Agradecimientos}

Este trabajo ha sido respaldado en parte por la administración pública de la Generalitat Valenciana bajo la beca ACIF/2018/106.

\section{Referencias}

ARGENTE, E; GARCIA-FORNES, A Y ESPINOSA, A, (2016) "Aplicando la metodología Flipped-Teaching en el Grado de Ingeniería Informática: una experiencia práctica" XXII Jenui, Almería, 6-8 de julio 2016.

ASIC - UPV "Guía de utilización de la Plantilla para la inserción de Unidades Didácticas para Microsoft Word 2010, 2013, 2015 y O365" < http://www,upv,es/entidades/ASIC/catalogo/U0838895,pdf $>$ [Consulta: 23 de marzo de 2020]

ASIC-ICE-UPV "Guía de implantación de la DOCENCIA INVERSA UPV" < http://www, upv,es/entidades/VRED/info/U0784192,pdf > [Consulta: 21 de marzo de 2020]

ASIC - Formación de doncencia inversa <http://docplayer,es/142356105-Formacio-n-proyecto-docenciainversa,html $>$ [Consulta: 20 de marzo de 2020]

CHIÑAS-PALACIOS, C; VARGAS-SALGADO, C, ÁGUILA-LEON, J; GARCIA E, (2019) "Zoom y Moodle: acortando distancias entre universidades, Una experiencia entre la Universidad de Guadalajara, México y la Universidad Libre de Colombia” IN-RED 2019: V Congreso de Innovación Educativa y Docencia en Red.

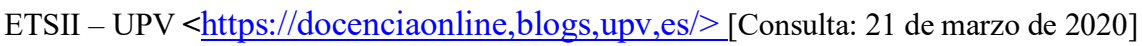


GOMEZ-TEJEDOR, J ; MOLINA MATEO, J ; SERRANO, MA ; MESEGUER DUEÑAS, J ; VIDAURRE, A ; RIERA, J, (2019) "Utilización de Lessons como herramienta de apoyo a la docencia inversa en la asignatura de Biofísica” IN-RED 2019: V Congreso de Innovación Educativa y Docencia en Red.

PEÑA, B; ZABALZA, I; LLERA,E Y USÓN, S, (2019) “El modelo de aula inversa en el área de Máquinas y Motores Térmicos: análisis y comparación de experiencias” IN-RED 2019: V Congreso de Innovación Educativa y Docencia en Red.

SÁNCHEZ CABALLERO, S, MONTAVA-JORDA, M,A, SELLES, A,V, MARTÍNEZ, (2019) "Implementación de las tareas semanales mediante la plataforma PoliformaT para la mejora de resultados en el aprendizaje por proyectos" IN-RED 2019: V Congreso de Innovación Educativa y Docencia en Red.

TERRASA, A, Y MARTÍNEZ, S (2020), "Planificación y desarrollo de mi proyecto de docencia inversa" DESIIC, ASIC-UPV

VI Jornada de Docencia Inversa en el Campus de Vera $<$ https://docenciainversa,blogs,upv,es/vi-jornadade-docencia-inversa-en-el-campus-de-vera/> [Consulta: 21 de marzo de 2020] 